\title{
Article
}

\section{Distance Fibonacci Polynomials by Graph Methods}

\author{
Dominik Strzałka $^{1}$ (D), Sławomir Wolski ${ }^{2}$ (D) and Andrzej Włoch ${ }^{2, *(\mathbb{D})}$ \\ 1 Faculty of Electrical and Computer Engineering, Rzeszów University of Technology, Aleja Powstańców \\ Warszawy 12, 35-959 Rzeszów, Poland; strzalka@prz.edu.pl \\ 2 Faculty of Mathematics and Applied Physics, Rzeszów University of Technology, Aleja Powstańców \\ Warszawy 12, 35-959 Rzeszów, Poland; wolan@prz.edu.pl \\ * Correspondence: awloch@prz.edu.pl
}

check for

updates

Citation: Strzałka, D.; Wolski, S.; Włoch, A. Distance Fibonacci Polynomials by Graph Methods. Symmetry 2021, 13, 2075. https:// doi.org/10.3390/sym13112075

Academic Editors: Jose M. Rodriguez and José M. Sigarreta

Received: 30 September 2021

Accepted: 20 October 2021

Published: 3 November 2021

Publisher's Note: MDPI stays neutral with regard to jurisdictional claims in published maps and institutional affiliations.

\begin{abstract}
In this paper we introduce and study a new generalization of Fibonacci polynomials which generalize Fibonacci, Jacobsthal and Narayana numbers, simultaneously. We give a graph interpretation of these polynomials and we obtain a binomial formula for them. Moreover by modification of Pascal's triangle, which has a symmetric structure, we obtain matrices generated by coefficients of generalized Fibonacci polynomials. As a consequence, the direct formula for generalized Fibonacci polynomials was given. In addition, we determine matrix generators for generalized Fibonacci polynomials, using the symmetric matrix of initial conditions.
\end{abstract}

Keywords: Fibonacci numbers; Fibonacci polynomials; matrix generators; Pascal's triangle

MSC: 11B39; 11B37; 11C20

\section{Introduction and Preliminary Results}

Let $k \geq 1, n \geq 0$ be integers. Sequences defined by the $(k+1)$-st order linear recurrence relation

$$
a_{n}=b_{1} a_{n-1}+b_{2} a_{n-2}+\cdots+b_{k+1} a_{n-(k+1)} \text {, for } n \geq k+1
$$

where $b_{i} \in N \cup\{0\}, i \in\{1,2, \ldots, k+1\}, b_{k+1} \neq 0$, with initial conditions $a_{n} \in N \cup\{0\}$, $n \in\{0,1, \ldots, k\}$, such that $\sum_{j=0}^{k} a_{j}^{2}>0$, are named sequences of the Fibonacci type and consequently their elements are Fibonacci type numbers.

For special cases of $k, b_{i}, i \in\{1,2, \ldots, k+1\}$ and $a_{n}, n \in\{0,1, \ldots, k\}$ the equality (1) gives the well-known number sequences. In this paper we consider the following sequences.

1. Fibonacci sequence $F_{n}=F_{n-1}+F_{n-2}$, for $n \geq 2$ and $F_{0}=0, F_{1}=1$.

2. Jacobsthal sequence $J_{n}=J_{n-1}+2 J_{n-2}$, for $n \geq 2$ and $J_{0}=0, J_{1}=1$.

3. Narayana sequence $N_{n}=N_{n-1}+N_{n-3}$, for $n \geq 3$ and $N_{0}=0, N_{1}=N_{2}=1$.

4. generalized Fibonacci sequence $F(p, n)=F(p, n-1)+F(p, n-p)$, for $p \geq 2$, $n \geq p+1$ and $F(p, n)=n+1$ for $n \in\{0, \ldots p-1\}$ (see for this concept [1]).

There are a number of other well-known Fibonacci type sequences, see for example [2].

Fibonacci sequence initiated a wide theory of Fibonacci type sequences. The recurrence which defines the sequence $\left(F_{n}\right)$ appears in 1202 in the book Liber Abaci of Italian mathematician Leonardo of Pisa, better known as Fibonacci, as a solution of the famous rabbit problem. This book was a compendium of the arithmetical and algebraic knowledge of those time and played an important role in the development of European mathematics. However, the name Fibonacci sequence for $\left(F_{n}\right)$ was introduced in the 19th century by $\mathrm{F}$. Lucas. It is worth mentioning that Fibonacci introduced the sequence $\left(F_{n}\right)$ to European mathematics, but that sequence was described earlier by Indian mathematics in works of Pingala and later Virachanka, see for details [3]. 
Fibonacci sequence has many interesting applications, for example, their connections with the golden ratio. Actually, there is a huge interest of modern science in the applications of golden section, which appears nowadays in physics research of the high energy particles and theoretical physics. Fibonacci numbers are intensively studied in different areas of science by mathematics enthusiasts and many interesting generalizations of Fibonacci sequence and Fibonacci type sequences have been defined. A special direction of generalization of the Fibonacci sequence is a sequence of Fibonacci polynomials $f_{n}(x)$, which were introduced by Catalan in 1883 and next studied with respect to their distinct properties, see, for examples, [4].

Fibonacci polynomials $f_{n}(x)$ are defined by the recurrence relation of the form

$$
f_{n}(x)=x f_{n-1}(x)+f_{n-2}(x), \text { for } n \geq 2
$$

with initial conditions $f_{0}(x)=0, f_{1}(x)=1$. Clearly $f_{n}(1)=F_{n}$.

Generalizations of numbers or polynomials of the Fibonacci type existing in the literature are mainly concentrated on combinatorial properties using generating functions or the problem of solving the recurrence relation, called a problem of Binet's formula type, see, for example, [5-7]. Note that some generalizations we obtain by changing initial terms and preserving the recurrence equation (see $[8,9]$ ) or by modification of the recurrence, see, for instance, [10].

In the literature we can also find another definition of Fibonacci polynomials which arises from the graph theory. A question of a graph interpretation of Fibonacci type numbers and polynomials is interesting not only from the graph theoretical point of view but also from their applications and the possibility to use graphs methods as a proving tools. Some results concerning applications of graphs in studying known sequences of the Fibonacci type were given, for example, in [11-17]. Motivated by their results in this paper we use graph methods to give properties of generalized Fibonacci polynomials. Let us first recall necessary graph concepts and give some historical background.

By a graph $G$ we mean a finite, undirected, connected, simple graph where $V(G)$ and $E(G)$ denote the vertex set and the edge set of $G$, respectively. By a $P_{n}$ we mean a graph with the vertex set $V\left(P_{n}\right)=\left\{t_{1}, \ldots, t_{n}\right\}$ and the edge set $E\left(P_{n}\right)=\left\{t_{i} t_{i+1} ; i \in\{1, \ldots, n-1\}\right\}$, $n \geq 2$. Moreover, $P_{0}$ is the empty graph and $P_{1}$ is the graph that consists of only one vertex. Let $K_{x}$ denote the complete graph on $x$ vertices, $x \geq 1$.

Let $G$ be a graph on $V(G)=\left\{v_{1}, \ldots, v_{n}\right\}, n \geq 2$ and $h_{n}=\left(H_{i}\right)_{i \in\{1, \ldots, n\}}$ be a sequence of vertex disjoint graphs on $V\left(H_{i}\right)=\left\{\left(v_{i}, y_{1}\right), \ldots,\left(v_{i}, y_{x}\right)\right\}, x \geq 1$. By the generalized lexicographic product of the graph $G$ and the sequence $h_{n}$ we mean the graph $G\left[h_{n}\right]$ such that $V\left(G\left[h_{n}\right]\right)=\bigcup_{i=1}^{n} V\left(H_{i}\right)$ and $E\left(G\left[h_{n}\right]\right)=\left\{\left(v_{i}, y_{p}\right)\left(v_{j}, y_{q}\right) ;\left(v_{i}=v_{j}\right.\right.$ and $\left(v_{i}, y_{p}\right)\left(v_{i}, y_{q}\right) \in$ $\left.E\left(H_{i}\right)\right)$ or $\left.v_{i} v_{j} \in E(G)\right\}$. If $H_{i}=H$ for $i \in\{1, \ldots, n\}$, then $G\left[h_{n}\right]=G[H]$, where $G[H]$ is the well-known composition of two graphs. By $H_{i}^{c}, i \in\{1, \ldots, n\}$ we will denote the copy of the graph $H_{i}$ in $G\left[h_{n}\right]$

Let $H$ be an arbitrary subgraph of $G\left[h_{n}\right]$. By a projection of a subgraph $H$ on the graph $G$ we mean a subgraph $p r_{G} H$ induced by the set $\{v \in V(G)$; there exists $1 \leq i \leq$ $n$ such that $\left.\left(v, y_{i}\right) \in V(H)\right\}$.

For $x, y \in V(G)$ by $d_{G}(x, y)$ we mean the distance between $x$ and $y$ in $G$.

A subset of vertices is independent if no two vertices are adjacent. Moreover the empty set and a set containing exactly one vertex also are independent.

The problem of counting of independent sets in graphs is related to the Fibonacci type numbers. Fibonacci numbers appears in graphs first in the paper of H. Prodinger and R. F. Tichy [18] in the context of counting independent sets in $n$-vertex path $P_{n}$. They proved that the total number of independent sets in $P_{n}$ is equal to $F_{n+2}$. This simple observation gave impetus for counting independent sets in graphs and finding graph interpretations of Fibonacci type numbers. The interest in counting independent sets in graphs was multiplied by the fact that the total number of independent sets in molecular graphs, named the Merrifield-Simmons index, has a huge application in combinational chemistry 
(see, for example, the survey [19] and its references). Based on the graph interpretation of Fibonacci numbers in 1983 G. Hopkins and W. Staton defined Fibonacci polynomials of graphs being the total number of independent sets in $G\left[K_{x}\right]$. In consequence of their results another type of Fibonacci polynomials can be defined as follows

$$
F_{n}(x)=F_{n-1}(x)+x F_{n-2}(x) \text { for } n \geq 2
$$

with initial conditions $F_{0}(x)=1$ and $F_{1}(x)=x+1$.

If $x=1$, then $F_{n}(1)=F_{n+2}$, see for details [20].

In spite of both $f_{n}(x)$ and $F_{n}(x)$ generalized Fibonacci numbers, $\left(f_{n}(x)\right)$ and $\left(F_{n}(x)\right)$ are different. For illustration let us observe the first 10 elements of these sequences.

$$
\begin{aligned}
& \left(f_{n}(x)\right):\left(0,1, x, x^{2}+1, x^{3}+2 x, x^{4}+3 x^{2}+1, x^{5}+4 x^{3}+3 x, x^{6}+5 x^{4}+6 x^{2}+1, x^{7}+6 x^{5}+\right. \\
& \left.10 x^{3}+4 x, x^{8}+7 x^{6}+15 x^{4}+10 x^{2}+1, x^{9}+8 x^{7}+21 x^{5}+20 x^{3}+5 x, \ldots\right) \\
& \left(F_{n}(x)\right):\left(1, x+1,2 x+1, x^{2}+3 x+1,3 x^{2}+4 x+1, x^{3}+6 x^{2}+5 x+1,4 x^{3}+10 x^{2}+6 x+1,\right. \\
& x^{4}+10 x^{3}+15 x^{2}+7 x+1,5 x^{4}+20 x^{3}+21 x^{2}+8 x+1, x^{5}+15 x^{4}+35 x^{3}+28 x^{2}+9 x+ \\
& \left.1,6 x^{5}+35 x^{4}+56 x^{3}+36 x^{2}+10 x+1, \ldots\right)
\end{aligned}
$$

Analogously, as Fibonacci numbers, Fibonacci polynomials $f_{n}(x)$ and $F_{n}(x)$ also have many generalizations and interpretations, see, for example, [21]. Some of them generalize Fibonacci polynomials in the distance sense, i.e., by changing the distance between terms of the sequence. Generalization of the Fibonacci polynomials $F_{n}(x)$ in the distance sense was given in 2003 by I. Włoch using graph methods, see for details [22].

As a consequence of results from [22] for $n \geq 0, k \geq 1$ generalized Fibonacci polynomials $F_{n}(k, x)$ can be defined by a recurrence relation.

$$
F_{n}(k, x)=F_{n-1}(k, x)+x F_{n-(k+1)}(k, x) \text { for } n \geq k+1
$$

with initial conditions $F_{0}(k, x)=1$ and $F_{n}(k, x)=n x+1$ for $n \in\{1, \ldots, k\}$. This sequence generalizes Fibonacci polynomials $F_{n}(1, x)=F_{n}(x)$ and also Fibonacci numbers, Jacobsthal numbers and Narayana numbers, since $F_{n}(1,1)=F_{n+2}, F_{n}(1,2)=J_{n+2}$ and $F_{n}(2,1)=$ $N_{n+3}$, respectively.

In this paper using graph methods we give a new distance generalization of Fibonacci polynomials. Existing definitions of Fibonacci type polynomials are given by putting the variable $x$ as a coefficient in the recurrence equation. In this paper we introduce another type of distance Fibonacci polynomial using the power of the variable $x$, which relates to the order of the recurrence equation. This implies that we can only use known methods partially.

Let $k \geq 1, n \geq 2$ be integers. Distance Fibonacci polynomials $\varphi_{n}(k, x)$ are defined by

$$
\varphi_{n}(k, x)=\varphi_{n-1}(k, x)+x^{k} \varphi_{n-(k+1)}(k, x) \text { for } n \geq k+1
$$

with initial conditions $\varphi_{n}(k, x)=1$ for $n \in\{0,1, \ldots, k-1\}$ and $\varphi_{k}(k, x)=1+x^{k}$.

Note that $\varphi_{n}(1, x)=F_{n}(x)$ and consequently $\varphi_{n}(1,1)=F_{n+1}, \varphi_{n}(1,2)=J_{n+1}$, $\varphi_{n}(2,1)=N_{n+2}$.

Theorem 1. Let $k \geq 1$ be an integer, $t$, and $R$ be real where $|t|<R, R>0$. The generating function for distance Fibonacci polynomials sequence $\left(\varphi_{n}(k, x)\right)$ is

$$
g(t, k)=\frac{1+x^{k} t^{k}}{1-t-x^{k} t^{k+1}} .
$$


Proof. Assume that the generating function has the form $g(t, k)=\sum_{n=0}^{\infty} \varphi_{n}(k, x) t^{n}$. Then

$$
\begin{aligned}
g(t, k) & =\sum_{n=0}^{\infty} \varphi_{n}(k, x) t^{n}=1+t+\cdots+t^{k-1}+\left(x^{k}+1\right) t^{k}+\sum_{n=k+1}^{\infty} \varphi_{n}(k, x) t^{n} \\
& =1+t+\cdots+t^{k-1}+\left(x^{k}+1\right) t^{k}+\sum_{n=k+1}^{\infty}\left(\varphi_{n-1}(k, x)+x^{k} \varphi_{n-(k+1)}(k, x)\right) t^{n} \\
& =1+t+\cdots+t^{k-1}+\left(x^{k}+1\right) t^{k}+\sum_{n=k+1}^{\infty} \varphi_{n-1}(k, x) t^{n}+\sum_{n=k+1}^{\infty} x^{k} \varphi_{n-(k+1)}(k, x) t^{n} \\
& =1+t+\cdots+t^{k-1}+\left(x^{k}+1\right) t^{k}+t \sum_{n=k+1}^{\infty} \varphi_{n-1}(k, x) t^{n-1} \\
& +x^{k} t^{k+1} \sum_{n=k+1}^{\infty} \varphi_{n-(k+1)}(k, x) t^{n-(k+1)} \\
& =1+t+\cdots+t^{k-1}+t^{k}+t^{k} x^{k}+t \sum_{n=k}^{\infty} \varphi_{n(k, x)} t^{n}+x^{k} t^{k+1} \sum_{n=0}^{\infty} \varphi_{n}(k, x) t^{n} \\
& =1+t^{k} x^{k}+t \sum_{n=0}^{\infty} \varphi_{n}(k, x) t^{n}+x^{k} t^{k+1} \sum_{n=0}^{\infty} \varphi_{n}(k, x) t^{n} \\
& =1+t^{k} x^{k}+t \cdot g(t, k)+x^{k} t^{k+1} \cdot g(t, k) .
\end{aligned}
$$

Hence we have $g(t, k)=1+x^{k} t^{k}+t \cdot g(t, k)+x^{k} t^{k+1} \cdot g(t, k)$ and $g(t, k)=\frac{1+x^{k} k^{k}}{1-t-x^{k} t^{k+1}}$.

For $k=1$ we have a generating function of the sequence of Fibonacci polynomials (3)

$$
g(t, x)=\frac{1+x t}{1-t-x t^{2}} .
$$

Moreover, for special values of $k$ and $x$ we obtain a generating function of Fibonacci, Jacobsthal and Narayana sequences, respectively.

$$
\begin{aligned}
& \sum_{n=0}^{\infty} F_{n+2} t^{n}=\frac{1+t}{1-t-t^{2}} \\
& \sum_{n=0}^{\infty} J_{n+2} t^{n}=\frac{1+2 t}{1-t-2 t^{2}} \\
& \sum_{n=0}^{\infty} N_{n+2} t^{n}=\frac{1+t^{2}}{1-t-t^{3}}
\end{aligned}
$$

Theorem 2. Let $n \geq 0, k \geq 1$ be integers. Then

$$
\sum_{i=0}^{n} \varphi_{i}(k, x)=\frac{\varphi_{n+k+1}(k, x)-1-x^{k}}{x^{k}} .
$$


Proof. (by induction on $n$ ). If $n=0$, then the equality (11) follows immediately. Assume that the formula (11) holds for $n, n \geq 1$. We shall show that it is also true for $n+1$.

$$
\begin{aligned}
\sum_{i=0}^{n+1} \varphi_{i}(k, x) & =\sum_{i=0}^{n} \varphi_{i}(k, x)+\varphi_{n+1}(k, x) \\
& =\frac{\varphi_{n+k+1}(k, x)-1-x^{k}}{x^{k}}+\frac{x^{k} \varphi_{n+1}(k, x)}{x^{k}} \\
& =\frac{\varphi_{n+k+1}(k, x)+x^{k} \varphi_{n+1}(k, x)-1-x^{k}}{x^{k}} \\
& =\frac{\varphi_{n+k+2}(k, x)-1-x^{k}}{x^{k}},
\end{aligned}
$$

which ends the proof.

\section{Graph Interpretation and Direct Formula of $\varphi_{n}(k, x)$}

We give a graph interpretation of polynomials $\varphi_{n}(k, x)$ with respect to counting of special induced subgraphs in a lexicographic product.

Let $H \leq G$ be an induced subgraph of $G$ such that every connected component of $H$ is isomorphic to $P_{k}$, for a fixed $k \geq 1$. In particular $H$ can be empty. A component of $H$ we will name the $P_{k}$-component and the subgraph $H$ with $p \geq 0$ components by the $p P_{k}$-subgraph.

Denote by $\mathcal{H}$ an induced subgraph of $G\left[h_{n}\right]$ satisfying conditions:

(a) each connected component of $\mathcal{H}$ is isomorphic to $P_{k}$,

(b) for each two vertices $\left(v, y_{i}\right),\left(v, y_{j}\right) \in V(\mathcal{H}) i=j$,

(c) $\quad p r_{G} \mathcal{H}$ is a $p P_{k}$-subgraph of $G, p \geq 0$.

In particular $\mathcal{H}$ can be empty.

Let $\varphi_{G\left[h_{n}\right]}(k, x)$ denote the number of all subgraphs $\mathcal{H}$ of $G\left[h_{n}\right]$.

Theorem 3. Let $k \geq 1, n \geq 0$ be integers. Then

$$
\varphi_{P_{n}\left[h_{n}\right]}(k, x)=\varphi_{n}(k, x) .
$$

Proof. (by induction on $n$ ) From the definition of $\mathcal{H}$ we have that at most one vertex from $H_{i}^{c}, i \in\{1, \ldots, n\}$ can belong to $\mathcal{H}$.

If $n \in\{0, \ldots, k-1\}$, then the empty set is the unique subgraph $\mathcal{H}$ of $P_{n}\left[h_{n}\right]$.

If $n=k$, then either $\mathcal{H}$ is empty or $\mathcal{H}$ contains exactly one $P_{k}$-component. Since the vertex from every $H_{i}^{c}$ can be chosen in $x$ ways, we have $x^{k}+1$ subgraphs $\mathcal{H}$, so $\varphi_{P_{k}\left[h_{k}\right]}(k, x)=x^{k}+1$.

Let $n \geq k+1$ and suppose that equality holds for each $t<n$. We shall show this for $n$. If $\left|V(\mathcal{H}) \cap V\left(H_{n}^{c}\right)\right|=0$, then $\mathcal{H} \cong \mathcal{H}^{*}$, where $\mathcal{H}^{*}$ is an induced subgraph of $P_{n-1}\left[h_{n-1}\right]$ satisfying conditions (a)-(c). Using the induction's hypothesis we have $\varphi_{n-1}(k, x)$ subgraphs $\mathcal{H}$.

If $\left|V(\mathcal{H}) \cap V\left(H_{n}^{c}\right)\right|=1$, then the $P_{k}$-component of $\mathcal{H}$ including the vertex from $H_{n}^{c}$ contains exactly one vertex from every $H_{i}^{c}, i \in\{n-(k-1), \ldots, n\}$. Since the vertex from $H_{i}^{c}, i \in\{n-(k-1), \ldots, n\}$, can be chosen in $x$ ways, so we have exactly $x^{k}$ such $P_{k}$-components. Moreover, $\mathcal{H}=\mathcal{H}^{\prime} \cup\left\{P_{k}\right\}$ where $\mathcal{H}^{\prime}$ is a subgraph of $P_{n-(k+1)}\left[h_{n-(k+1)}\right]$ satisfying conditions (a)-(c). In particular $\mathcal{H}^{\prime}$ can be empty. So by the induction's hypothesis we have $x^{k} \varphi_{n-(k+1)}(k, x)$ subgraphs $\mathcal{H}$.

From the above we have that $\varphi_{P_{n}\left[h_{n}\right]}(k, x)=\varphi_{n-1}(k, x)+x^{k} \varphi_{n-(k+1)}(k, x)=\varphi_{n}(k, x)$ and by the induction rule the theorem is proved.

Now we determine the direct formula for $\varphi_{n}(k, x)$ using the above graph interpretation.

Let $k \geq 1, n \geq 0, p \geq 0$ be integers. By $\sigma_{G}(n, k, p)$ we denote the total number of $p P_{k}$-subgraphs of a graph $G$. 
Theorem 4 . Let $k \geq 1, n \geq 0, x \geq 1$ be integers. Then for an arbitrary graph $G$ on $n$ vertices

$$
\varphi_{G\left[h_{n}\right]}(k, x)=\sum_{p \geq 0} \sigma_{G}(k, n, p) x^{k p} .
$$

Proof. Let $G$ be a given graph on $n$ vertices. If $n \in\{0, \ldots, k-1\}$ the result is obvious. Let $n \geq k$. To determine the number $\varphi_{G\left[h_{n}\right]}(k, x)$ firstly we have to choose a $p P_{k}$-subgraph of the graph $G$. Clearly we can choose a $p P_{k}$-subgraph in $\sigma_{G}(k, n, p)$ ways. Let $S=$ $\left\{v_{i} ; i \in \mathcal{I} \subset\{1, . ., p k\}\right\}$ be a vertex set of a $p P_{k}$-subgraph of $G$. Let $S^{\prime} \subset V\left(G\left[h_{n}\right]\right)$ and $S^{\prime}=\left\{\left(v_{i}, y_{t}\right) ; v_{i} \in S\right.$ and $\left.1 \leq t \leq x\right\}$. In other words, for each $H_{i}^{c}$ such that $v_{i} \in S$ we choose exactly one vertex from $V\left(H_{i}^{c}\right)$ to the set $S^{\prime}$. Clearly this vertex can be chosen in $x$ ways. From the definition of the $G\left[h_{n}\right]$ we have that $S^{\prime}$ is a subgraph $\mathcal{H}$ satisfying conditions (a)-(c) with $p P_{k}$-components. Since each $P_{k}$-component from $S$ generates $x^{k}$ possible components $\mathcal{H}$, we obtain $\sigma_{G}(k, n, p) x^{k p}$ subsets $\mathcal{H}$ of $G\left[h_{n}\right]$. So we have $\sum_{p \geq 0} \sigma_{G}(k, n, p) x^{k p}$ subgraphs $\mathcal{H}$, which ends the proof.

Lemma 1. Let $k \geq 1, n \geq 0, p \geq 0$ be integers. Then

$$
\sigma_{P_{n}}(k, n, p)=\left(\begin{array}{c}
n-p k+1 \\
p
\end{array}\right)
$$

Proof. Consider a path $P_{n}$ with $V\left(P_{n}\right)=\left\{v_{1}, \ldots, v_{n}\right\}$ and vertices are numbered in the natural fashion. If $n \in\{0, \ldots, k-1\}$, then the result is obvious. Let $n \geq k$ and $H$ be a $p P_{k}$-subgraph of $P_{n}$.

If $H=\varnothing$, then the result immediately follows.

Let $H \neq \varnothing$. Let $\alpha(v)$ be the function such that $\alpha(v)=1$ if $v \in V(H)$ or $\alpha(v)=0$ otherwise. For convenience for every $v_{i} \in V\left(P_{n}\right)$ we will write shortly $\alpha_{i}$ instead of $\alpha\left(v_{i}\right)$. Then instead of a graph $P_{n}$ we can consider a binary $n$-tuple. Clearly a $P_{k}$-component of $H$ corresponds to a subsequence $\beta$ of $k$ consecutive $1 \mathrm{~s}$ in the $n$-tuple $\alpha=\left(\alpha_{1}, \ldots \alpha_{n}\right)$. To determine the number $\sigma_{P_{n}}(k, n, p)$ it suffices to consider all binary $n$-tuples $\left(\alpha_{1}, \ldots \alpha_{n}\right)$ such that $\sum_{i=1}^{n} \alpha_{i}=p k$ and satisfying additional conditions given below.

Denote by $\left(\beta_{1}, \ldots, \beta_{p}\right), p \geq 1$ sequence of $k$-tuples such that

(i) every $\beta_{j}, 1 \leq j \leq p$ contains $k$ s being consecutive in the sequence $\alpha$,

(ii) if $\alpha_{i} \in \beta_{j}, 1 \leq i \leq n, 1 \leq j \leq p$, then $\alpha_{i}=1$,

(iii) for each two $\beta_{j}, \beta_{r}$ there is a subsequence of $n$-tuple $\left(\alpha_{1}, \ldots \alpha_{n}\right)$ of the form $\left(\beta_{j}, 0, \beta_{r}\right)$.

We substitute each $\beta_{j}, 1 \leq j \leq p$, by exactly one 1 and obtain $(n-p(k-1))$-tuple containing $p$ non-consecutive 1 s. To determine all possible $(n-p(k-1))$-tuples firstly we consider $(n-p k)$-tuple of zeros and next we put $p 1$ s using $n-p k+1$ places, so $\sigma_{P_{n}}(k, n, p)=\left(\begin{array}{c}n-p k+1 \\ p\end{array}\right)$.

$$
\text { Let } \sigma_{G}(k, n)=\sum_{p \geq 0} \sigma_{G}(k, n, p)
$$

Corollary 1 . Let $k \geq 1, n \geq 0, p \geq 0$ be integers. Then

$$
\sigma_{P_{n}}(k, n)=\sum_{p \geq 0}\left(\begin{array}{c}
n-p k+1 \\
p
\end{array}\right) .
$$

From the Theorems 3 and 4 and Lemma 1 we obtain the binomial formula for $\varphi_{n}(k, x)$.

Corollary 2 . Let $k \geq 1, n \geq 0, x \geq 1$ be integers. Then

$$
\varphi_{n}(k, x)=\varphi_{P_{n}\left[h_{n}\right]}(k, x)=\sum_{p \geq 0}\left(\begin{array}{c}
n-p k+1 \\
p
\end{array}\right) x^{k p} .
$$




\section{Connections with Pascal's Triangle}

It is well known that Fibonacci numbers can be obtained from Pascal's triangle. Many authors studying Fibonacci type sequences also give connections with Pascal's triangle or modified Pascal's triangle, see [23-25]. Such interpretations can be used to derive binomial formulas for Fibonacci type sequences. In [26] it was proved that all Fibonacci type sequences have binomial formulas. It is natural to find similar properties of generalized distance Fibonacci polynomials. In this section we inspect infinite matrices generated by coefficients of generalized distance Fibonacci polynomials. These matrices we can obtain by simple modifications of Pascal's triangle.

For a convenience we will write Pascal's triangle in a matrix form.

$$
P=\left[\begin{array}{llllllllllll}
1 & 0 & 0 & 0 & 0 & 0 & 0 & 0 & 0 & 0 & 0 & \ldots \\
1 & 1 & 0 & 0 & 0 & 0 & 0 & 0 & 0 & 0 & 0 & \ldots \\
1 & 2 & 1 & 0 & 0 & 0 & 0 & 0 & 0 & 0 & 0 & \ldots \\
1 & 3 & 3 & 1 & 0 & 0 & 0 & 0 & 0 & 0 & 0 & \ldots \\
1 & 4 & 6 & 4 & 1 & 0 & 0 & 0 & 0 & 0 & 0 & \ldots \\
1 & 5 & 10 & 10 & 5 & 1 & 0 & 0 & 0 & 0 & 0 & \ldots \\
1 & 6 & 15 & 20 & 15 & 6 & 1 & 0 & 0 & 0 & 0 & \ldots \\
1 & 7 & 21 & 35 & 35 & 21 & 7 & 1 & 0 & 0 & 0 & \ldots \\
1 & 8 & 28 & 56 & 70 & 56 & 28 & 8 & 1 & 0 & 0 & \ldots \\
1 & 9 & 36 & 84 & 126 & 126 & 84 & 36 & 9 & 1 & 0 & \ldots \\
1 & 10 & 45 & 120 & 210 & 252 & 210 & 120 & 45 & 10 & 1 & \ldots \\
\vdots & \vdots & \vdots & \vdots & \vdots & \vdots & \vdots & \vdots & \vdots & \vdots & \vdots & \ldots \\
& & & & & & & & & & &
\end{array}\right]
$$

Let us consider for example generalized distance Fibonacci polynomials for $k=1$, $k=2$ and $k=3$. Initial polynomials of these sequences are:

$$
\begin{aligned}
& \varphi_{n}(1, x): 1, x+1,2 x+1, x^{2}+3 x+1,3 x^{2}+4 x+1, x^{3}+6 x^{2}+5 x+1,4 x^{3}+10 x^{2}+6 x+ \\
& 1, x^{4}+10 x^{3}+15 x^{2}+7 x+1,5 x^{4}+20 x^{3}+21 x^{2}+8 x+1, x^{5}+15 x^{4}+35 x^{3}+28 x^{2}+ \\
& 9 x+1, \ldots \\
& \varphi_{n}(2, x): 1,1, x^{2}+1,2 x^{2}+1,3 x^{2}+1, x^{4}+4 x^{2}+1,3 x^{4}+5 x^{2}+1,6 x^{4}+6 x^{2}+1, x^{6}+10 x^{4}+ \\
& 7 x^{2}+1,4 x^{6}+15 x^{4}+8 x^{2}+1,10 x^{6}+21 x^{4}+9 x^{2}+1, x^{8}+20 x^{6}+28 x^{4}+10 x^{2}+1,5 x^{8}+ \\
& \quad 35 x^{6}+36 x^{4}+11 x^{2}+1, \ldots \\
& \varphi_{n}(3, x): 1,1,1, x^{3}+1,2 x^{3}+1,3 x^{3}+1,4 x^{3}+1, x^{6}+5 x^{3}+1,3 x^{6}+6 x^{3}+1,6 x^{6}+7 x^{3}+ \\
& 1,10 x^{6}+8 x^{3}+1, x^{9}+15 x^{6}+9 x^{3}+1,4 x^{9}+21 x^{6}+10 x^{3}+1,10 x^{9}+28 x^{6}+11 x^{3}+ \\
& 1, \ldots
\end{aligned}
$$

If we write coefficients of consecutive polynomials in rows of a matrix, then an element $a_{i, j}$ is the coefficient at $x^{(j-1) k}$. The matrices $A_{1}$ and $A_{2}$ presented below correspond to sequences for $k=1$ and 2 , respectively.

$$
A_{1}=\left[\begin{array}{ccccccccc}
1 & 0 & 0 & 0 & 0 & 0 & 0 & 0 & 0 \\
1 & 1 & 0 & 0 & 0 & 0 & 0 & 0 & 0 \\
1 & 2 & 0 & 0 & 0 & 0 & 0 & 0 & 0 \\
1 & 3 & 1 & 0 & 0 & 0 & 0 & 0 & 0 \\
1 & 4 & 3 & 0 & 0 & 0 & 0 & 0 & 0 \\
1 & 5 & 6 & 1 & 0 & 0 & 0 & 0 & 0 \\
1 & 6 & 10 & 4 & 0 & 0 & 0 & 0 & 0 \\
1 & 7 & 15 & 10 & 1 & 0 & 0 & 0 & 0 \\
1 & 8 & 21 & 20 & 5 & 0 & 0 & 0 & 0 \\
1 & 9 & 28 & 35 & 15 & 1 & 0 & 0 & 0 \\
1 & 10 & 36 & 56 & 35 & 6 & 0 & 0 & 0 \\
1 & 11 & 45 & 84 & 70 & 21 & 1 & 0 & 0 \\
1 & 12 & 55 & 120 & 126 & 56 & 7 & 0 & 0 \\
1 & 13 & 66 & 165 & 210 & 126 & 28 & 1 & 0 \\
1 & 14 & 78 & 220 & 330 & 252 & 84 & 8 & 0
\end{array}\right] \quad A_{2}=\left[\begin{array}{cccccc}
1 & 0 & 0 & 0 & 0 & 0 \\
1 & 0 & 0 & 0 & 0 & 0 \\
1 & 1 & 0 & 0 & 0 & 0 \\
1 & 2 & 0 & 0 & 0 & 0 \\
1 & 3 & 0 & 0 & 0 & 0 \\
1 & 4 & 1 & 0 & 0 & 0 \\
1 & 5 & 3 & 0 & 0 & 0 \\
1 & 6 & 6 & 0 & 0 & 0 \\
1 & 7 & 10 & 1 & 0 & 0 \\
1 & 8 & 15 & 4 & 0 & 0 \\
1 & 9 & 21 & 10 & 0 & 0 \\
1 & 10 & 28 & 20 & 1 & 0 \\
1 & 11 & 36 & 35 & 5 & 0 \\
1 & 12 & 45 & 56 & 15 & 0
\end{array}\right]
$$


Let $C_{i}$ denote the ith column of the matrix $P$. We obtain a matrix $A_{k}$, for an arbitrary $k \geq 1$, from the matrix $P$ by the following transformation: The column $C_{2}$ we shift $k-1$ rows downward and columns $C_{i}, i \geq 3$, we shift $(i-1) k-1$ rows downward. See, for example, matrices $A_{1}$ and $A_{2}$.

We can observe that sums in rows or in diagonals form known sequences, see [27], dependences are presented in Table 1.

Table 1. Sums in rows and sums on diagonals of $A_{k}$ matrices.

\begin{tabular}{cccc}
\hline$k$ & $A_{\boldsymbol{k}}$ & Sums in Rows of $\boldsymbol{A}_{\boldsymbol{k}}$ & Sums on Diagonals of $\boldsymbol{A}_{\boldsymbol{k}}$ \\
\hline 1 & $A_{1}$ & Fibonacci sequence & Narayana sequence \\
\hline 2 & $A_{2}$ & Narayana sequence & A003269 [27] \\
\hline 3 & $A_{3}$ & A003269 [27] & \\
\hline
\end{tabular}

Analogously to classical Pascal's triangle we can give a general rule of calculating entries of a matrix $A_{k}$. For a convenience, in the next theorem rows and columns of a matrix are numbered starting from the number zero.

Theorem 5. Let $k \geq 1, n \geq 0, i \geq 0$ be integers. Then $A_{k}=\left[a_{n, i}\right]$ where $a_{n, i}=a_{n-1, i}+a_{n-(k+1), i-1}$ with $a_{n, 0}=1$ for $n \in\{0, \ldots, k-1\}$ and $a_{n, i}=0$ for $n<0$ or $i<0$.

Proof. For $i \in\{1, \ldots k\}$ the result is obvious.

Let $i>k$. Then $\varphi_{n}(k, x)=\sum_{i=0}^{\infty} a_{n, i} i^{i k}$ where coefficients $a_{n, i}$ are elements of the matrix $A_{k}$. Then

$$
\begin{aligned}
\varphi_{n}(x) & =\varphi_{n-1}(k, x)+x^{k} \varphi_{n-(k+1)}(k, x)=\sum_{i=0}^{\infty} a_{n-1, i} x^{i k}+\sum_{i=0}^{\infty} a_{n-(k+1), i} x^{i k+k} \\
& =\sum_{i=0}^{\infty}\left(a_{n-1, i} x^{i k}+a_{n-(k+1), i} x^{i k+k}\right)=\sum_{i=0}^{\infty}\left(a_{n-1, i} x^{i k}+a_{n-(k+1), i-1} x^{i k}\right) \\
& =\sum_{i=0}^{\infty}\left(a_{n-1, i}+a_{n-(k+1), i-1}\right) x^{i k} .
\end{aligned}
$$

Comparing coefficients at $x^{n}$ we have $a_{n, i}=a_{n-1, i}+a_{n-(k+1), i-1}$, which ends the proof.

We can also calculate polynomials $\varphi_{n}(k, x)$ directly from Pascal's triangle $P$. This method we will call a staircase method.

$$
P=\left[\begin{array}{lllllllllll}
1 & 0 & 0 & 0 & 0 & 0 & 0 & 0 & 0 & 0 & 0 \\
1 & 1 & 0 & 0 & 0 & 0 & 0 & 0 & 0 & 0 & 0 \\
1 & 2 & 1 & 0 & 0 & 0 & 0 & 0 & 0 & 0 & 0 \\
1 & 3 & 3 & 1 & 0 & 0 & 0 & 0 & 0 & 0 & 0 \\
1 & 4 & 6 & 4 & 1 & 0 & 0 & 0 & 0 & 0 & 0 \\
1 & 5 & 10 & 10 & 5 & 1 & 0 & 0 & 0 & 0 & 0 \\
1 & 6 & 15 & 20 & 15 & 6 & 1 & 0 & 0 & 0 & 0 \\
1 & 7 & 21 & 35 & 35 & 21 & 7 & 1 & 0 & 0 & 0 \\
1 & 8 & 28 & 56 & 70 & 56 & 28 & 8 & 1 & 0 & 0 \\
1 & 9 & 36 & 84 & 126 & 126 & 84 & 36 & 9 & 1 & 0 \\
1 & 10 & 45 & 120 & 210 & 252 & 210 & 120 & 45 & 10 & 1
\end{array}\right]
$$

For example, for $k=2$ we have stairs with steps of the height equal to 2 and the length equal to 1 . We extend the stairs presented above up to infinity in both directions. Moving such infinite stairs one row downward we obtain coefficients of consecutive polynomials. The blue colour indicates coefficients of $\varphi_{9}(2, x)$ and the red colour coefficients of $\varphi_{8}(3, x)$. is $k$.

Generally for an arbitrary $k \geq 1$ the length of the step is 1 and the height of the step 
The above considerations lead to the same binomial formula for $\varphi_{n}(k, x)$ as in Corollary 2.

Theorem 6. Let $k \geq 1, n \geq 0$ and $x \geq 1$ be integers. Then

$$
\varphi_{n}(k, x)=\sum_{i=0}^{\left\lfloor\frac{n}{k}\right\rfloor}\left(\begin{array}{c}
n+1-i k \\
i
\end{array}\right) x^{i k}
$$

Proof. Let $a_{n, i}^{k}$ denote an element of a matrix $A_{k}$. We know that $\varphi_{n}(k, x)=\sum_{i=0}^{n} a_{n, i}^{k} x^{i k}$. To prove this theorem it is enough to perform transformations $A_{k}$ to Pascal's triangle $P$. A reverse operation to shifting columns downwards gives $a_{n, i}^{k}=p_{n-i k, i}$ and we obtain a corresponding entry in Pascal's triangle $P$. In the sum we omit zero terms, because all entries above the main diagonal of Pascal's triangle are zeros. Thus we have

$$
\varphi_{n}(k, x)=\sum_{i=0}^{\left\lfloor\frac{n}{k}\right\rfloor}\left(\begin{array}{c}
n+1-i k \\
i
\end{array}\right) x^{i k}
$$

which ends the proof.

\section{Matrix Generators}

Linear recurrence equations have interesting relations with matrices. Matrix generators of Fibonacci type sequences were used to describe properties of these sequences, see, for example, $[4,28,29]$. We show that matrix generators of $\varphi_{n}(k, x)$ can be found using the method given in $[21,28]$.

Let $k \geq 1$ be an integer and $Q_{k}=\left[q_{i j}\right]_{(k+1) \times(k+1)}$ be a square matrix. For a fixed $1 \leq i \leq k+1$ an element $q_{i, 1}$ is equal to the coefficient at $\varphi_{n-i}(x)$ of the right hand side of the formula (5). If $j>1$, then $q_{i, j}=\left\{\begin{array}{ll}1 & j=i+1 \\ 0 & \text { otherwise }\end{array}\right.$. The above definition gives matrices $Q_{1}=\left[\begin{array}{ll}1 & 1 \\ x & 0\end{array}\right], Q_{2}=\left[\begin{array}{ccc}1 & 1 & 0 \\ 0 & 0 & 1 \\ x^{2} & 0 & 0\end{array}\right], Q_{3}=\left[\begin{array}{cccc}1 & 1 & 0 & 0 \\ 0 & 0 & 1 & 0 \\ 0 & 0 & 0 & 1 \\ x^{3} & 0 & 0 & 0\end{array}\right], \ldots$, $Q_{k}=\left[\begin{array}{ccccc}1 & 1 & 0 & \cdots & 0 \\ 0 & 0 & 1 & \cdots & 0 \\ \vdots & \vdots & \vdots & \ddots & \vdots \\ 0 & 0 & 0 & \cdots & 1 \\ x^{k} & 0 & 0 & \cdots & 0\end{array}\right]$

To obtain a matrix generator of $\varphi_{n}(k, x)$, firstly, we define a square matrix $R_{k}$ of order $k+1$ as the matrix of initial conditions

$$
R_{k}=\left[\begin{array}{cllcc}
\varphi_{2 k}(k, x) & \varphi_{2 k-1}(k, x) & \cdots & \varphi_{k+1}(k, x) & \varphi_{k}(k, x) \\
\varphi_{2 k-1}(k, x) & \varphi_{2 k-2}(k, x) & \cdots & \varphi_{k}(k, x) & \varphi_{k-1}(k, x) \\
\vdots & \vdots & \ddots & \vdots & \vdots \\
\varphi_{k-1}(k, x) & \varphi_{k}(k, x) & \cdots & \varphi_{2}(k, x) & \varphi_{1}(k, x) \\
\varphi_{k}(k, x) & \varphi_{k-1}(k, x) & \cdots & \varphi_{1}(k, x) & \varphi_{0}(k, x)
\end{array}\right]
$$

Using basic properties of determinants, we get the following results.

Theorem 7. Let $k \geq 1$ be an integer. Then

$$
\operatorname{det} Q_{k}=(-1)^{k} x^{k} \text { and } \operatorname{det} R_{k}=(-1)^{\left\lfloor\frac{k+1}{2}\right\rfloor} x^{k^{2}+k} \text {. }
$$


Theorem 8. Let $k \geq 1, n \geq 1$ be integers. Then

$$
R_{k} Q_{k}^{n}=\left[\begin{array}{ccccc}
\varphi_{n+2 k}(k, x) & \varphi_{n+2 k-1}(k, x) & \cdots & \varphi_{n+k+1}(k, x) & \varphi_{n+k}(k, x) \\
\varphi_{n+2 k-1}(k, x) & \varphi_{n+2 k-2}(k, x) & \cdots & \varphi_{n+k}(k, x) & \varphi_{n+k-1}(k, x) \\
\vdots & \vdots & \ddots & \vdots & \vdots \\
\varphi_{n+k+1}(k, x) & \varphi_{n+k}(k, x) & \cdots & \varphi_{n+2}(k, x) & \varphi_{n+1}(k, x) \\
\varphi_{n+k}(k, x) & \varphi_{n+k-1}(k, x) & \cdots & \varphi_{n+1}(k, x) & \varphi_{n}(k, x)
\end{array}\right] .
$$

Proof. (by induction on $n$ ) If $n=1$, then by (5) the result immediately follows. Assume that formula (19) holds for $n$; we will prove it for $n+1$. Since $R_{k} Q_{k}^{n+1}=\left(R_{k} Q_{k}^{n}\right) Q_{k}$, by our assumption and by the recurrence (5) we obtain $R_{k} Q_{k}^{n+1}=$

$$
\begin{gathered}
{\left[\begin{array}{ccccc}
\varphi_{n+2 k}(k, x) & \varphi_{n+2 k-1}(k, x) & \cdots & \varphi_{n+k+1}(k, x) & \varphi_{n+k}(k, x) \\
\varphi_{n+2 k-1}(k, x) & \varphi_{n+2 k-2}(k, x) & \cdots & \varphi_{n+k}(k, x) & \varphi_{n+k-1}(k, x) \\
\vdots & \vdots & \ddots & \vdots & \vdots \\
\varphi_{n+k+1}(k, x) & \varphi_{n+k}(k, x) & \cdots & \varphi_{n+2}(k, x) & \varphi_{n+1}(k, x) \\
\varphi_{n+k}(k, x) & \varphi_{n+k-1}(k, x) & \cdots & \varphi_{n+1}(k, x) & \varphi_{n}(k, x)
\end{array}\right]\left[\begin{array}{ccccc}
1 & 1 & 0 & \cdots & 0 \\
0 & 0 & 1 & \cdots & 0 \\
\vdots & \vdots & \vdots & \ddots & \vdots \\
0 & 0 & 0 & \cdots & 1 \\
x^{k} & 0 & 0 & \cdots & 0
\end{array}\right]} \\
=\left[\begin{array}{ccccc}
\varphi_{n+2 k+1}(k, x) & \varphi_{n+2 k}(k, x) & \cdots & \varphi_{n+k+2}(k, x) & \varphi_{n+k+1}(k, x) \\
\varphi_{n+2 k}(k, x) & \varphi_{n+2 k-1}(k, x) & \cdots & \varphi_{n+k+1}(k, x) & \varphi_{n+k}(k, x) \\
\vdots & \vdots & \ddots & \vdots & \vdots \\
\varphi_{n+k+2}(k, x) & \varphi_{n+k+1}(k, x) & \cdots & \varphi_{n+3}(k, x) & \varphi_{n+2}(k, x) \\
\varphi_{n+k+1}(k, x) & \varphi_{n+k}(k, x) & \cdots & \varphi_{n+2}(k, x) & \varphi_{n+1}(k, x)
\end{array}\right],
\end{gathered}
$$

which ends the proof.

By Theorem 7 we can obtain the following result, it being the Cassini formula for Fibonacci polynomials $\varphi_{n}(k, x)$.

Corollary 3. Let $k \geq 3, n \geq 2$ be integers. Then

$$
\operatorname{det} R_{k} Q_{k}^{n}=(-1)^{n k+\left\lfloor\frac{k+1}{2}\right\rfloor} x^{k^{2}+(n+1) k} .
$$

\section{Conclusions}

Sequences of the Fibonacci type defined by the homogenous linear recurrence relations can be extended to polynomials. In the literature we have found distinct definitions of Fibonacci type polynomials. Most of them are obtained by putting the variable $x$ as a coefficient in the recurrence equation of the Fibonacci type sequences. It is necessary to pay attention to the connection of the Fibonacci type polynomials with counting problems in graphs. Graph interpretations of Fibonacci type polynomials give useful tools in studying properties of that sequence, in particular in finding direct formulas.

As introduced in this paper, distance Fibonacci polynomials by graph interpretation in the lexicographic product of graphs could initiate the study of other Fibonacci type polynomials with respect to graph methods. It seems to be interesting to study other Fibonacci type polynomials which generalize, for example, Lucas, Pell, Padovan numbers with respect to graph interpretations.

Author Contributions: Conceptualization, D.S., S.W. and A.W.; methodology, D.S., S.W. and A.W.; validation, D.S., S.W. and A.W.; formal analysis, D.S., S.W. and A.W.; investigation, D.S., S.W. and A.W.; writing-original draft preparation, D.S., S.W. and A.W.; writing-review and editing, D.S., S.W. and A.W.; visualization, D.S., S.W. and A.W. All authors have read and agreed to the published version of the manuscript.

Funding: This research received no external funding.

Institutional Review Board Statement: Not applicable. 
Informed Consent Statement: Not applicable.

Data Availability Statement: Not applicable.

Conflicts of Interest: The authors declare no conflict of interest.

\section{References}

1. Kwaśnik, M.; Włoch, I. The Total Number of Generalized Stable Sets and Kernels in Graphs. Ars Comb. 2000, 55, 139-146.

2. Szynal-Liana, A.; Włoch, I. Hypercomplex Numbers of the Fibonacci Type; Oficyna wydawnicza Politechniki Rzeszowskiej: Rzeszów, Poland, 2019.

3. Parmanand, S. So-Call. Fibonacci Numbers Anc. Mediev. India. Historia Math. 1985, 12, 229-244.

4. Koshy, T. Fibonacci and Lucas Numbers with Applications; John Wiley and Sons: New York, NY, USA, 2001.

5. Kilic, E. The Binet formula, sums and representations of generalized Fibonacci p-numbers. Eur. J. Comb. 2008, 29, 701-711. [CrossRef]

6. Stakhov, A.; Rozin, B. Theory of Binet formulas for Fibonacci and Lucas p-numbers. Chaos Solitons Fractals 2006, $27,1162-1177$. [CrossRef]

7. Trojovský, P. On the characteristic polynomial of (k,p)-Fibonacci sequence. Adv. Differ. Equ. 2021, 2021. [CrossRef]

8. Panwar, Y.K.; Singh, B.; Gupta V.K. Generalized Fibonacci Polynomials. Turk. J. Anal. Number Theory 2013, 1, 43-47. [CrossRef]

9. Singh, M.; Sikhwal, O.; Parsai, V.; Gupta, Y.K. Generalized Fibonacci-Lucas Polynomials. Int. J. Adv. Math. Sci. 2014, 2, 81-87. [CrossRef]

10. Taştan, M.; Özkan, E.; Shannon, A.G. The generalized k-Fibonacci polynomials and generalized k-Lucas polynomials. Notes Number Theory Discret. Math. 2021, 27, 148-158. [CrossRef]

11. Kalman, D. Generalized Fibonacci numbers by matrix methods. Fibonacci Quart. 1982, 20, 73-76.

12. Startek, M.; Włoch, A.; Włoch, I. Fibonacci numbers and Lucas numbers in graphs. Discret. Appl. Math. 2009, 157, 864-868. [CrossRef]

13. Benjamin, A.; Yerger, C. Combinatorial Interpretations of Spanning Tree Identities. Bull. Inst. Comb. Its Appl. .2006, 47, 37-42.

14. Kılıç, E.; Stakhov, A. On the Fibonacci and Lucas p-numbers, their sums, families of bipartite graphs and permanents of certain matrices. Chaos Solitons Fractals 2009, 40, 2210-2221. [CrossRef]

15. Włoch, I. On generalized Pell numbers and their graph representations. Comment. Math. 2008, 48, 169-175.

16. Bród, D. On a two-parameter generalization of Jacobsthal numbers and its graph interpretation. Ann. Univ. Mariae CurieSklodowska Sect. A 2018, 72, 21-28. [CrossRef]

17. Yilmaz, F.; Bozkurt, D. The Adjacency Matrix of One Type of Directed Graph and the Jacobsthal Numbers and Their Determinantal Representation. J. Appl. Math. 2012, 2012, 423163. [CrossRef]

18. Prodinger, H.; Tichy, R.F. Fibonacci numbers of graphs. Fibonacci Quart. 1982, 20, 16-21.

19. Gutman, I.S. Wagner, S. Maxima and minima of the Hosoya index and the Merrifield-Simmons index. A survey of results and techniques. Acta Appl. Math. 2010, 112, 323-346.

20. Hopkins, G.; Staton, W. Some identities arising from the Fibonacci numbers of certain graphs. Fibonacci Quart. 1984, 22, 255-258.

21. Bednarz, U.; Wołowiec-Musiał, M. Distance Fibonacci Polynomials. Symmetry 2020, 12, 1540. [CrossRef]

22. Włoch, I. Generalized Fibonacci polynomial of graph. Ars Comb. 2003, 68, 49-55.

23. Falcón, S.; Plaza, Á. The k-Fibonacci sequence and the Pascal 2-triangle. Chaos Solitons Fractals 2007, 33, 38-49. [CrossRef]

24. Matoušová, I.; Trojovský, P. On Coding by (2,q)-Distance Fibonacci Numbers. Mathematics 2020, 8, 2058. [CrossRef]

25. Kuhapatanakul, K. The Fibonacci p-numbers and Pascal's triangle. Cogent Math. 2016, 3, 1264176. [CrossRef]

26. Włoch, I.; Włoch, A. On some multinomial sums related to the Fibonacci type numbers. Tatra Mt. Math. Publ. 2020, 77, 99-108. [CrossRef]

27. Sloane, N.J.A. The On-Line Encyclopedia of Integer Sequences. Available online: https:/ / oeis.org/ (accessed on 25 September 2021).

28. Bednarz, U.; Włoch, A.; Wołowiec-Musiał, M. Distance Fibonacci numbers, their interpretations and matrix generators. Comment. Math. 2013, 53, 35-46. [CrossRef]

29. Dasdemir, A. On the Pell, Pell-Lucas and modified Pell numbers by matrix method. Appl. Math. Sci. 2011, 5, 3173-3181 